\title{
Global Issues of Food Production
}

\section{Ali Ghasemzadeh*}

Department of Crop Science, University Putra Malaysia, Selangor, Malaysia

The major driving force for increased food, feed, fibre and biofuel production is global population growth, which translates into an increase of about 30 million people per year, mostly in developing countries. Achieving global food and nutrition security for 9.2 billion people by 2050 which means that food production will need to be increased by $70 \%$ from current levels in a sustainable way while the environmental footprint of agriculture is reduced. Small holder farmers in developing countries are expected to meet the food demand of emerging rural and urban regions. This demand is likely to increase to almost $90 \%$ of total food demand by 2050 . Worldwide, an additional 1 billion tonnes of cereals and 200 million tonnes of meat will need to be produced annually. Farmers must therefore, have access to new and innovative technologies and adopt intensive farming practices using the principles of global nutrient stewardship. The increase in production will have to be realized with a decreasing rural labour force and limited land, water and energy resources, while mitigating and adapting to climate change (Figure 1).

FAO (2009) anticipates that $90 \%$ of the growth in crop production globally will come from higher yields and increased cropping intensity, with the remainder coming from land expansion. Cereal yield growth would continue to slow down, to some $0.7 \%$ annually, and average cereal yield would increase from 3.2 to 4.3 tonnes per hectare by 2050 . Natural resources, particularly water supply, will be outstripped by demand. UNDP (2006) has predicted that over 3 billion people are likely to experience water stress by 2025 , a very serious constraint on agricultural growth and development, especially in droughtprone areas. Land will be limited due to competition for housing and industrial uses, while the opening up of new land for agriculture is not environmentally sustainable. Arable land will have expanded by some 70 million hectares (Mha) at the global level in 2050, with expansion in developing countries of about 120 Mha being offset by a decline of some 50 Mha in developed countries. Almost all of the land expansion in developing countries would take place in Sub-Saharan Africa and Latin America. Since 1961, at the time of the Green Revolution, total food, animal feed and livestock output has almost trebled, representing an average increase of $2.3 \%$ per year. During the 20th century, the composition of agricultural production started to change in order to meet the diet diversification of the growing urban population and rising incomes. Production of meat, fruits and vegetables and oil crops accelerated, driven by growth in demand in developing countries and by biofuel use in many developed countries. Demand for bioenergy increases in synergy with petroleum prices. Bioenergy demand is creating new markets for agricultural products that can be used as

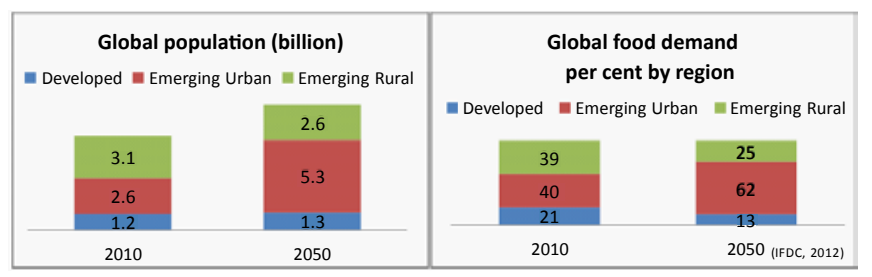

Figure 1: Comparing of global population and food demand percent by region at 2010 and 2050. feedstock for the production of biofuels. The implications of bioenergy development on food security and poverty alleviation deserve increased attention and further analysis. The toll of climate change on agricultural production and food security, particularly in tropical areas, is becoming increasingly clear. Policies and investments supporting trade, sustainable agricultural practices and technological progress can help mitigate the negative effects of climate change.

\section{Future Actions for Sustainable Agricultural Production}

Global agricultural production should focus on both food and nutrition security. Ensuring that, sufficient nutritious food is available and accessible, and produced in a sustainable manner, is a great challenge for the agricultural sector. Output will need to be increased while managing scarce natural resources, reducing carbon intensity and adverse environmental impacts throughout the food chain, enhancing the provision of environmental services such as carbon sequestration and flood and drought control, and conserving biodiversity. Agricultural intensification is the answer, but it must be economically, socially and environmentally sustainable. Higher use efficiency of fertilizers and other nutrient sources can maintain or improve soil fertility and increase yields while minimizing environmental impacts. However, world agriculture is dominated by smallholders, who find it difficult to keep up with rising demand for food, feed, fibre and biofuels. Extensive farming systems are still widespread, particularly in Sub-Saharan Africa, where the gap between average national yields and average yields observed in demonstration plots is significant. Expanding access to inputs, and to existing technologies and knowledge, would make it possible to quickly reduce the yield gap in these countries. Providing the right source of plant nutrients where and when they are needed by farmers will be the focus of the fertilizer industry. Fertilizer use increases almost in synchrony with cereal yield, while the crop area decreases (Figure 2).

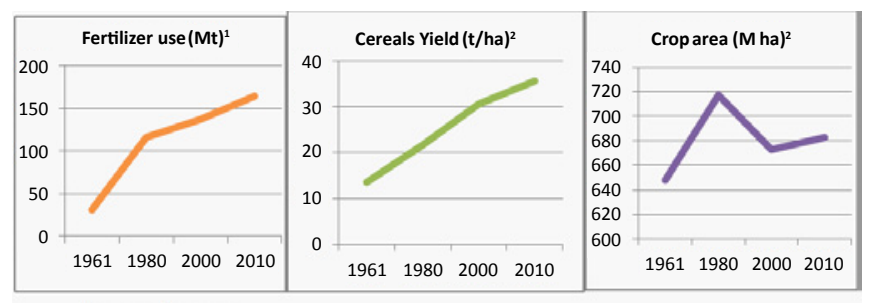

Source: ${ }^{1}$ IFADATA: ${ }^{2}$ FAOSTAT

Figure 2: Fertilizer use, Cereals yield and Crop area Since 1961 to 2010.

*Corresponding author: Ali Ghasemzadeh, Department of Crop Science University Putra Malaysia, Selangor, Malaysia, E-mail: upmali@yahoo.com

Received September 07, 2012; Accepted September 09, 2012; Published September 16, 2012

Citation: Ghasemzadeh A (2012) Global Issues of Food Production. Agrotechnol 1:e102. doi:10.4172/2168-9881.1000e102

Copyright: (c) 2012 Ghasemzadeh A. This is an open-access article distributed under the terms of the Creative Commons Attribution License, which permits unrestricted use, distribution, and reproduction in any medium, provided the original author and source are credited. 


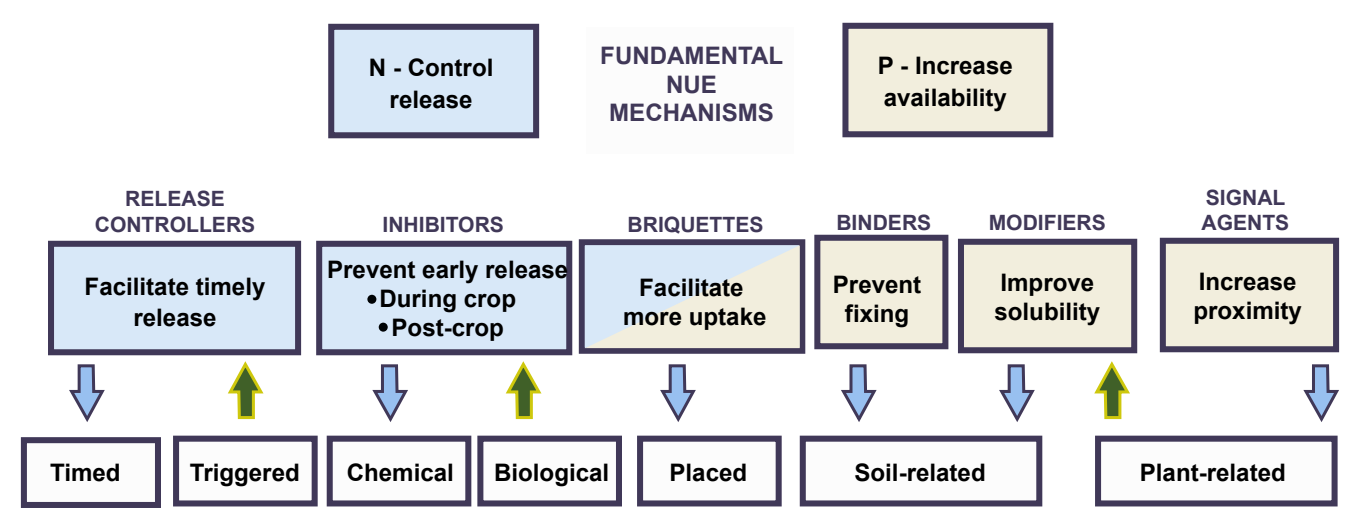

Figure 3: Nutrient use efficiency.

Global fertilizer consumption reached 173 million tonnes nutrients in 2010/11, with nitrogen dominating this demand at 104.5 million tonnes. East and South Asia, Latin America and Central Europe contributed largely to this demand growth (Table 1). Tentative demand forecasts for $2012 / 13$ point to $2.3 \%$ growth, reaching $182.2 \mathrm{Mt}$ as agricultural commodity prices are seen to remain attractive but highly volatile during the period.

East and South Asia will contribute largely to the increase in global fertilizer demand, by 1.7 and $1.1 \mathrm{Mt}$, respectively (IFA, 2011). Increasing nutrient use efficiency (in particular, that of nitrogen and phosphorus) is of high importance in many mature markets to maximize benefits and minimize the environmental footprint. In response, the fertilizer industry should develop a new generation of intelligent fertilizers that minimize economic and environmental drawbacks and ensure maximum crop production. Producing this next generation of intelligent fertilizers with improved efficiency, increased convenience and accuracy in delivering secondary and micronutrients, effective and efficient sourcing and delivery processes that will lessen cost and the risk of crop failure for smallholder farmers, while reducing environmental impact, will be the industry's future direction. Improvements or alternatives to current production methods will need to be introduced, and the cost and environmental impact of fertilizer use reduced, with efficiency considerations for developing countries that are dominated by smallholder farmers (Figure 3 ).

Innovative approaches to effective knowledge transfer in agriculture and best management practices will be needed to maximize the benefits of agricultural inputs. Advances in biotechnology, particularly with respect to improved crops adapted to climate and weather changes, with higher yields and more efficient utilization of nutrients, among other traits, will no doubt contribute to increased food production. Improvements in the efficiency of irrigation and water management are also essential for future agricultural development. Overall, investment in agriculture needs to increase substantially, focusing on innovation, research and effective knowledge transfer to improve agriculture's role as an engine of growth and poverty reduction so that it can function as a longer-term pillar for global food and nutrition security. 